\title{
FATORES DETERMINANTES DA ADESÃO DE HIPERTENSOS A CONDUTA DIETOTERÁPICA
}

\section{DETERMINANT FACTORS OF THE ADHESION OF HYPERTENSIVES TO DIET-THERAPIC CONDUCT}

\author{
Lais Tatiele Massing ${ }^{\mathrm{a}^{*}}$, Marilene Rodrigues Portella $\mathrm{a}^{\mathrm{b}^{* *}}$

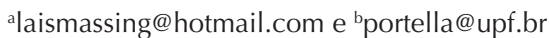 \\ *Pós-Graduação Universidade de Passo Fundo - Passo Fundo (RS), Brasil \\ ** Docente Universidade de Passo Fundo - Passo Fundo (RS), Brasil
}

Data de entrada do artigo: 07/12/2013

Data de aceite do artigo: 08/05/2014

\section{RESUMO}

Introdução: No Brasil, a hipertensão é um grave problema para a saúde pública. Cerca de 17 milhôes de brasileiros são hipertensos, dos quais $35 \%$ são adultos com 40 anos ou mais. Objetivo: $\mathrm{O}$ estudo objetivou avaliar os fatores determinantes da adesão de hipertensos a uma conduta dietoterápica. Metodologia: Trata-se de um estudo exploratório descritivo, de abordagem qualitativa, realizado em um município do noroeste do Rio Grande do Sul, com 95 adultos e idosos cadastrados como hipertensos no programa Estratégia Saúde na Família I (ESF I). A amostra foi do tipo não probabilística, e os dados foram coletados por meio da técnica de seminário; na organização deste levaram-se em conta tópicos para discussão em pequenos grupos, com exposição na plenária final para validação. Para análise dos dados, utilizou-se a técnica da análise temática, cujos resultados permitem inferir que os hipertensos têm conhecimento sobre a conduta dietoterápica recomendada como terapêutica não farmacológica. Resultados: Os motivos apontados como determinantes da falta de cuidado no controle da hipertensão são fatores intrínsecos, como as características pessoais de negação da doença, teimosia, compulsão e negligência; como fatores extrínsecos, foram apontadas a situação financeira e a dificuldade de acesso ao alimento integral. Discussão: A cronicidade dessa enfermidade gera um impacto muito grande na vida das pessoas e impóe adaptaçóes. Os fatores culturais influenciam o modo de agir frente às situaçóes de saúde ou doença. Conclusão: $\mathrm{O}$ estudo permitiu observar o conhecimento dos pacientes quanto ao tratamento não medicamentoso $\mathrm{e}$ notou a importância de novas estratégias de controle da hipertensão.

Palavras-chave: hipertensão; dietoterapia; adesão.

\section{ABSTRACT}

Introduction: In Brazil, hypertension is a serious problem for public health. Approximately 17 million of Brazilians are hypertensive, from which $35 \%$ are adults of 40 years or more. Object: The study had the objective to evaluate the factors determinants of the adhesion to a hypertensive diet-therapic conduct. Methods: This is an exploratory descriptive study of qualitative approach accomplished in a town of the northwest of Rio Grande do Sul state, with ninety five adults and elderly registered as hypertensive in the Strategy Health in the Family Program (SHF). The sample was not probabilistic and the data were collected through seminary. In the organization of the seminary it was taken into account topics for discussion in small groups with exposition at the final plenary for validation. To analyze the data it was used the technique of the thematic analyses, whose results allowed us to conclude that hypertensives have knowledge about the diet-therapic procedure recommended as non-pharmacological therapeutic. Results: The reasons cited as determinants of the lack of care in hypertension control are intrinsic factors, such as the personal characteristics of negation of disease, stubbornness, compulsion and negligence; as extrinsic 
factor were mentioned the financial situation and the difficulty of access to wholesome food. Discussion: The chronicity of this infirmity causes a great impact on the lives of these people and imposes adaptations. The cultural factors are strongly related to how to act when facing-situations of health or disease. Conclusion: This study allowed us to observe the knowledge of the patient about the nonon-pharmacological treatment, and it noticed the importance of new strategies to control hypertension.

Keywords: Nursing, Health Promotion, Mental Health.

\section{Introdução}

A hipertensão arterial sistêmica (HAS) é uma doença que envolve múltiplos fatores e é caracterizada por níveis elevados de pressão arterial $(\mathrm{PA})^{1}$. Essa condição clínica é associada frequentemente a alteraçóes estruturais ou funcionais de órgãos como: coração, encéfalo, rins e vasos sanguíneos, que podem sofrer alteraçōes metabólicas, as quais geram um risco aumentado no acometimento de problemas cardiovasculares fatais ou não fatais ${ }^{2}$.

A patologia é definida como pressão arterial sistólica (PAS) maior ou igual a $140 \mathrm{mmHg}$ e uma pressão arterial diastólica (PAD) maior ou igual a $90 \mathrm{mmHg}$ em indivíduos adultos, sem uso de medicação anti-hipertensiva. A HAS pode dividir-se em estágio 1, no qual a PAS oscila entre 140 a $159 \mathrm{mmHg}$ e o PAD, entre 90 a $99 \mathrm{mmHg}$, e estágio 2, com PAS maior que $160 \mathrm{mmHg}$ e PAD maior que $100 \mathrm{mmHg}^{3}$.

De acordo com a Organização Pan-Americana, a hipertensão bem como o diabetes são os principais responsáveis pelas internaçóes hospitalares e diálises, consequentemente, geram altos custos ao Sistema Único de Saúde (SUS); também são as principais doenças que levam a primeira causa de mortalidade no país ${ }^{4}$.

No Brasil e no mundo, a HAS é um grave problema para a saúde pública. Cerca de 17 milhôes de brasileiros são hipertensos, dos quais 35\% são adultos com 40 anos ou mais; e sabe-se que esse número está aumentando e atingindo a população mais jovem, inclusive crianças ${ }^{3}$.

Em estudo realizado em quinze capitais brasileiras e Distrito Federal através de inquérito telefônico, observou-se que a frequência de hipertensão autorreferida aumentou de acordo com a idade ${ }^{5}$. No que concerne à escolaridade, a pesquisa constatou que pessoas com ensino fundamental incompleto apresentaram prevalência em relação aos indivíduos que possuíam ensino fundamental completo. Entre os gêneros, constatou-se prevalência entre homens até 50 anos, invertendo-se o quadro após essa idade 5 .

A hipertensão não tem cura, mas o tratamento previne complicações. A orientaçáo alimentar realizada pelo nutricionista deve ser encorajada, já que é um dos fatores importantes do tratamento náo farmacológico, que tem como principal objetivo diminuir a morbidade e mortalidade cardiovascular ${ }^{6}$.
Para pacientes que possuem pressão arterial limítrofe ou baixo risco para doenças cardiovasculares, indica-se tratamento náo medicamentoso isolado durante seis meses ou até o aparecimento de lesão de órgãos-alvo; após esse período, se a pressão não estiver controlada, inicia-se o tratamento farmacológico em conjunto ${ }^{7}$.

$\mathrm{O}$ tratamento não medicamentoso consiste em medidas de controle que envolvem: eliminação do consumo de álcool e tabaco, prática de atividade física regularmente, perda do excesso de peso e modificação dos hábitos alimentares por meio da dietoterapia ${ }^{8}$.

A conduta dietoterápica tem como objetivo a perda e manutençáo do peso e deve ser realizada por meio de alteraçōes no estilo de vida, com ênfase em reeducação alimentar associada a atividade física. Essa ferramenta traduz-se na prescrição de um plano alimentar individualizado segundo guias de recomendaçóes nutricionais e cardápios diários, com sugestôes de substituição por equivalentes calóricos? ${ }^{9}$.

A orientação nutricional do paciente consiste em carboidratos complexos, fibras, proteínas, frutas, verduras, leite e derivados desnatados, água e baixo teor de gordura ${ }^{11}$. Essas mudanças podem ser uma das causas da não persistência no tratamento nutricional ${ }^{10}$. Esse tipo de alimentação favorece o suprimento de cálcio, potássio e magnésio, responsáveis pelo controle da PA. A suplementação só é recomendada nas situaçôes de hipocalcemia e hipomagnesemia ${ }^{3}$.

São escassas as referências acerca da suplementação de cálcio e magnésio como medida preventiva da hipertensão; encontra-se artigos que citam essa suplementação na dieta de acordo com as recomendaçōes das $V I$ Diretrizes Brasileiras de Hipertensáo Arterial ${ }^{3}$.

A suplementação de potássio pode promover uma pequena redução na hipertensão arterial, e a sua recomendação para pessoas sem diminuição da função renal é de $4,7 \mathrm{~g} /$ dia, porém é necessário cautela no uso desse suplemento em indivíduos que fazem uso de medicamentos como inibidores da ECA, em antagonistas do receptor ATI, em diuréticos poupadores de potássio e em pacientes com insuficiência renal suscetíveis a hiperpotassemia. Esse mineral auxilia na inibição da formação de radicais livres nas células dos vasos, bem como na inibição da agregação plaquetária e trombose arterial, aumenta a filtração glomerular e diminui a resistência vascular renal ${ }^{12}$. $\mathrm{O}$ consumo de potássio pode ser 
aumentado através da ingestão de feijão, ervilha, vegetais de cor verde-escuro, banana, melão, cenoura, beterraba, frutas secas, tomate, batata inglesa e laranja, além de alimentos pobres em sódio².

A perda de peso é outro fator indispensável, pois a relação do aumento de peso com a pressão é linear; assim, a perda de peso traz uma melhora na regulação da pressão e no metabolismo ${ }^{13}$. A perda de dez quilos pode diminuir a pressão arterial sistólica de cinco a $20 \mathrm{mmHg}^{14}$.

A atividade física regular é recomendada, pois, além de ajudar na perda de peso, diminui a PA e os riscos cardiovasculares e a mortalidade em geral. É considerada prática regular quando praticada trinta minutos por dia em pelo menos cinco dias da semana, com intensidade moderada de forma que possa ser realizada mantendo-se a conversaçãa ${ }^{3}$.

Quanto ao tabagismo, o risco para HAS aumenta gradualmente conforme o número de cigarros e a profundidade da inalação. Mulheres fumantes apresentam maior chance de complicaçóes do que homens. Assim como o tabaco, o álcool está relacionado com a HAS; por isso, as VI Diretrizes Brasileiras de Hipertensão Arterial limitam a ingestão em $30 \mathrm{ml} /$ dia para homens e $15 \mathrm{ml} /$ dia para mulheres ${ }^{3}$.

Caso o tratamento não farmacológico não obtenha os resultados esperados no controle da HAS, inicia-se o uso de anti-hipertensivos, como objetivo de reduzir os níveis tensionais.

De acordo com as VI Diretrizes Brasileiras de Hipertensão Arterial, o tratamento com esse tipo de medicação deve garantir eficácia por via oral, ser bem tolerado pelo paciente, permitir o menor número possível de administração diária e iniciar o tratamento com doses pequenas, aumentando de acordo com a necessidade $^{13}$. Os anti-hipertensivos possuem cinco classes, divididas de acordo com as distintas formas de sua ação terapêutica na fisiopatologia da doença ${ }^{13}$.

Não há um entendimento único no que se refere à classificação; verificam-se estudos que citam o uso de seis classes de anti-hipertensivos, que são os diuréticos, betabloquadores, simpatolíticos de ação central, antagonistas do canal de cálcio, inibidores da enzima conversora e antagonistas do receptor de angiotensina II. Ainda de acordo com o autor, no período inicial do tratamento farmacológico, é indicado o uso da monoterapia com doses reduzidas, aumentando gradativamente a dose combinada ou não com outra droga, e, se necessário, é indicada a substituição de medicação, dependendo da condição clínica do paciente ${ }^{12,13}$.

Para que o tratamento da hipertensão tenha uma evolução positiva, é necessário a adesão do paciente a uma conduta dietoterápica, que consiste nas modificaçôes alimentares a partir de escolhas mais saudáveis para melhora e manutençáo de sua saúde. Segundo a Organização Mundial da Saúde (OMS), a adesão está ligada ao comportamento de um indivíduo e é representada pela ingestáo de medicação, obediência à dieta, mudança no estilo de vida, correspondência e concordância com as demais recomendaçôes do profissional de saúde ${ }^{15}$.

Um estudo avaliou a adesão de pacientes a terapia nutricional e observou que $68,8 \%$ dos indivíduos desistiram do tratamento alegando a dificuldade na mudança dos hábitos alimentares como a principal causa da não adesão ${ }^{10}$.

Abaixa adesão ao tratamento é o principal fator que gera o baixo controle da HAS. De acordo com a pesquisa, cerca de $40 \%$ a $60 \%$ dos hipertensos não fazem uso da medicação, e esses valores aumentam ainda mais quando há relação com mudanças no estilo de vida e alimentaçãoo ${ }^{16}$. Fatores que implicam em mudanças nos hábitos, com a alteração de costumes prazerosos como alimentação excessiva, fumo, uso de bebidas alcoólicas e ócio, se tornam frustrantes para pessoas que não têm um apoio adequado e, por conseguinte favorecem a não-adesão ${ }^{10}$. Outro determinante pode estar relacionado à demora no agendamento da consulta em serviços públicos ${ }^{17}$.

Diante disso, o presente estudo teve como objetivo avaliar os fatores determinantes da adesão de hipertensos a conduta dietoterápica, visando a melhoria da qualidade de vida desses pacientes por meio de estratégias de prevenção dos fatores de riscos associados e de promoção da saúde.

\section{Metodologia}

O estudo foi realizado em um município de pequeno porte da regiấo noroeste do Rio Grande do Sul, com aproximadamente 3.978 habitantes de origens cabocla, italiana e alemã. Trata-se de um estudo exploratório descritivo, de abordagem qualitativa, com hipertensos cadastrados no programa Estratégia Saúde da Família I (ESF I), tomando por referência o mês de agosto de 2013. A amostra foi do tipo não probabilística, seleção esta que corresponde aos objetivos do estudo.

Foram considerados participantes do estudo adultos e idosos que atenderam aos critérios de inclusão: estar cadastrado na ESF I com condição de hipertenso, ter idade superior a 19 anos, livre adesão, cognição preservada, com capacidade de compreensão e em condiçóes de participação de uma atividade que exige a expressão verbal, e ser residente no município.

Os dados foram coletados por meio da técnica de seminário. Esse procedimento permitiu que os participantes debatessem sobre a temática e validassem na 
plenária final. Por meio de um convite com ampla divulgação, se determinou o dia para o "seminário" e, na referida ocasiáo, depois de detalharem minuciosamente os objetivos e o propósito do estudo, para aqueles que aderiram à proposta, firmou-se o consentimento por meio da assinatura do Termo de Consentimento Livre e Esclarecido (TCLE). Em seguida, os participantes foram convidados a se reunir em grupos de no máximo 15 pessoas de acordo com o sexo e a faixa etária (menores de 50 anos, entre 50 e 60 anos e maiores de 60 anos); em cada grupo, participou um agente comunitário de saúde (ACS) com o propósito de registrar as anotações e as ideais principais que emergiram durante a discussão.

A pesquisadora fez a abertura do seminário reforçando mais uma vez o propósito do encontro; feito isso, lançou as seguintes questóes para serem debatidas nos grupos: 1) O que sabemos sobre os cuidados que o hipertenso deve ter com a alimentação para combater pressão alta ou controlar a pressão? 2) Quando um hipertenso não cuida da sua alimentação, quais serão os motivos para essa falta de cuidado?

Durante as discussóes, o papel do ACS foi de estimular o debate e registrar as respostas. Feita a discussáo nos grupos, abriu-se espaço para a exposiçáo e leitura dos resultados dos trabalhos de cada grupo. Nesse momento, o ACS relatou os pontos que o grupo discutiu, e a pesquisadora registrou a síntese. As respostas na forma de síntese foram transcritas em um papel pardo (papel Kraft), em letra legível para que os participantes pudessem acompanhar. No final da exposiçấo, foi feita a leitura da síntese geral do que apareceu nos grupos, e, neste momento, a plenária também se manifestou validando e/ou fazendo comentários acerca dos dados.

Para análise e interpretação dos dados, utilizou-se a análise de conteúdo, mais especificamente a técnica da análise temática proposta por Minayo ${ }^{18}$, com as três etapas: a) pré-análise, que engloba a escolha dos documentos que foram analisados, retomando os objetivos iniciais da pesquisa, reformulando-os e orientando a interpretaçáo final momento que novamente se tomaram todos os relatos dos grupos e as sínteses da plenária final para a leitura flutuante, da constituição dos corpos, na formulação de hipóteses e objetivos; b) exploração de material, isto é, a transformaçáo dos dados brutos visando o alcance da compreensão do texto, abstraindo os núcleos de significado momento em que o material foi submetido a análise com proposição de inferências e interpretaçóes tomando por base o referencial construído; c) construção das duas temáticas "Conhecimento sobre recomendações dietéticas no controle da hipertensão" e "Motivos apontados para a falta de cuidado no controle da hipertensão".
A pesquisadora foi responsável pelo treinamento e pela supervisáo dos agentes comunitários de saúde que participaram do seminário, na condiçáo de relatora e facilitadores dos grupos. O treinamento inicial teve duração de duas horas para apresentação do projeto de pesquisa, ocasião em que também foi feita uma simulação das atividades do dia. No grupo de ACSs, usou-se a mesma dinâmica do dia do seminário. $\mathrm{O}$ treinamento foi realizado uma semana antes da data marcada para a realização do seminário.

Este estudo, nos seus aspectos éticos, observou as diretrizes da Resoluçáo no 466/2012 do Conselho Nacional de Saúde ${ }^{19}$ sobre pesquisa com seres humanos e foi submetido ao Comitê de Ética em Pesquisa da Universidade de Passo Fundo, obtendo aprovação por meio do processo $\mathrm{n}^{\mathrm{o}} 330.116$.

Estiveram presentes no seminário 107 hipertensos cadastrados no programa Estratégia Saúde da Família I no município estudado, porém, destes, somente 95 assinaram o Termo de Consentimento Livre e Esclarecido e participaram da pesquisa, sendo 70 do sexo feminino e 25 do sexo masculino; os demais que decidiram não fazer parte do seminário formaram um grupo de ouvintes.

Os hipertensos foram divididos em dez grupos de acordo com o sexo e a idade: um grupo de mulheres de até 50 anos, com 14 integrantes; um grupo de homens de até 50 anos com somente 3 homens integrantes; dois grupos de mulheres de 50 a 60 anos com 9 e 10 integrantes; um grupo de homens de 50 a 60 anos, com 5 integrantes; três grupos de mulheres de 60 anos ou mais, dois deles com 12 e o outro com 13 integrantes; dois grupos de homens de 60 anos, um com 10 e o outro com 7 integrantes.

\section{Resultados}

De acordo com os grupos, o conhecimento dos hipertensos sobre as recomendaçóes dietéticas no controle da hipertensão está descrito na Figura 1, subdivididas em cinco grupos referentes a: preferências, limitaçóes, restrições, práticas e crenças.

$\mathrm{Na}$ Figura 2, estão descritos os motivos apontados pelos participantes como determinantes da falta de cuidado, ou seja, as razóes pelas quais os hipertensos não seguem as recomendaçôes dietoterápicas para o controle da hipertensão. Os motivos estão divididos por fatores intrínsecos que envolvem características e questóes pessoais, fatores extrínsecos, que abarcam processos econômicos. 
Figura 1: Conhecimento dos participantes sobre recomendações dietéticas adotadas no controle da hipertensão.

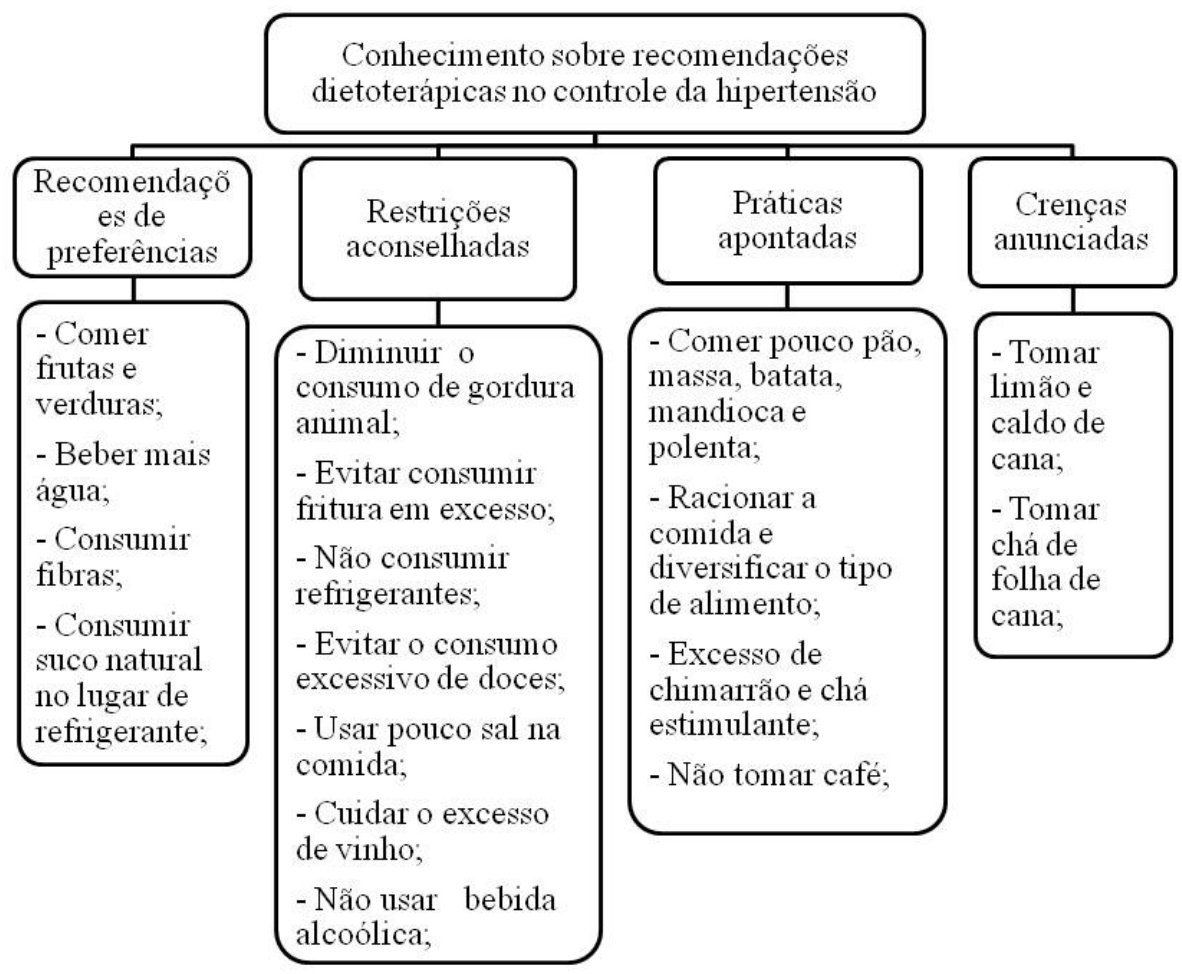

Figura 2: Motivos apontados como determinantes da falta de cuidado no controle da hipertensão.

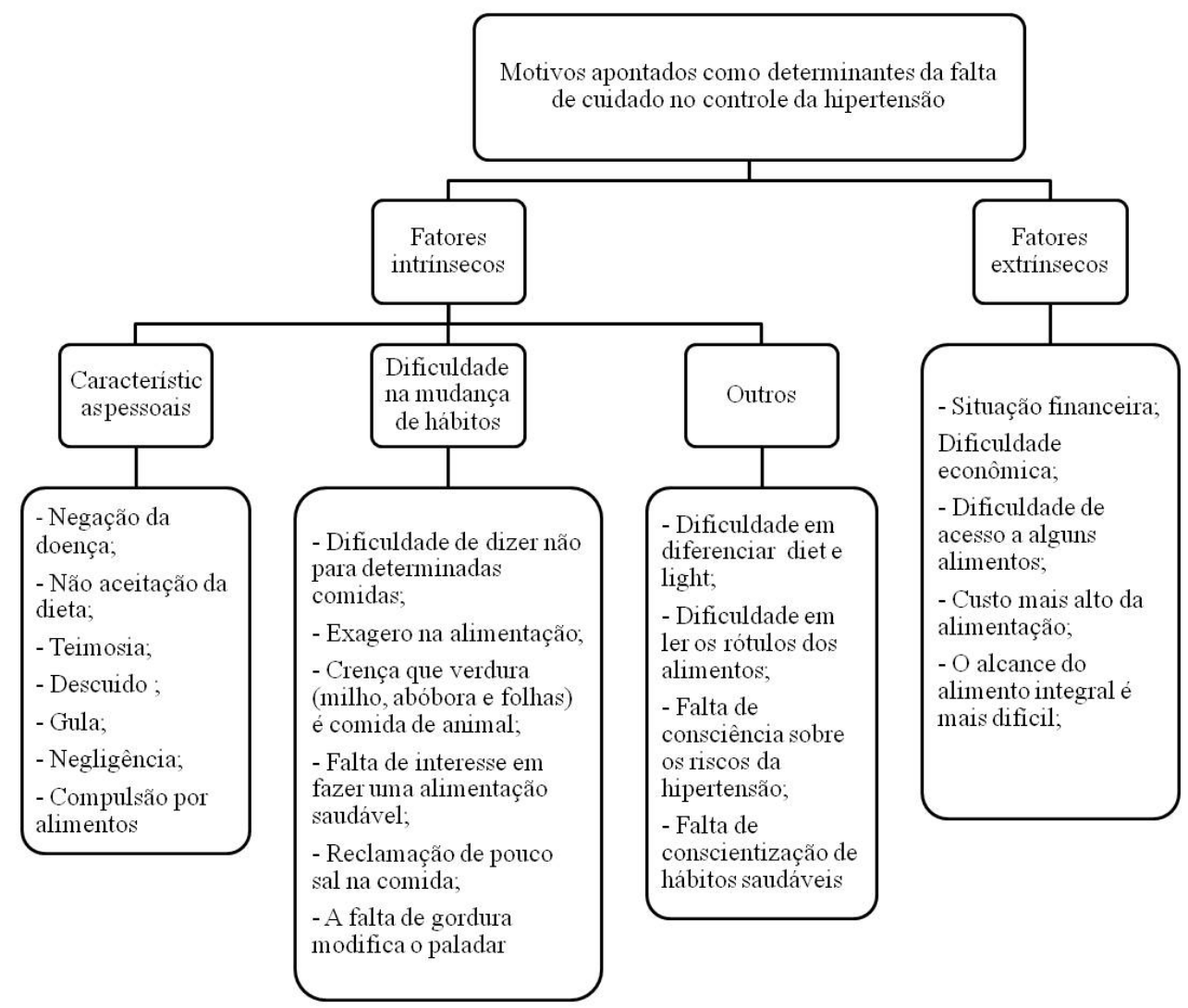




\section{Discussão}

A hipertensão destaca-se por sua relação com o risco de desenvolver doenças cardiovasculares como acidente vascular cerebral e infarto agudo do miocárdio e por sua relevância epidemiológica de alta prevalência ${ }^{20}$.

No ponto de vista dos portadores, essa patologia é compreendida de maneira particular, pois envolve uma gama de sentimentos, simbologias e preocupaçóes. A cronicidade dessa enfermidade gera um impacto muito grande na vida dessas pessoas e impóe adaptaçôes no dia a dia, levando a mudanças significativas e necessárias em suas práticas de vida ${ }^{20}$.

Fatores culturais estão fortemente relacionados ao modo de agir das pessoas frente a situações de saúde ou doença. E, por este motivo, as práticas de vida e o cuidado desses indivíduos serão orientados pela combinação da cultura e por suas vivências, condiçôes físicas e emocionais e motivação pessoal ${ }^{20}$.

De acordo com as VI Diretrizes Brasileiras de Hipertensão Arterial, a alimentação do hipertenso deve ser rica em frutas, hortaliças, fibras, minerais e laticínios com baixos teores de gordura ${ }^{13}$. Estudo cita mudanças relatadas pela grande maioria, na reduçáo do consumo de alimentos de risco, sendo as restriçóes no consumo de sal $(84,2 \%)$, gorduras $(36,2 \%)$ e doces $(26,0 \%)$ as mais referidas; o autor presume que os indivíduos associam o controle da hipertensão à diminuição no consumo desses produtos, e não ao consumo de alimentos protetores, como frutas, verduras e legumes ${ }^{21}$.

$\mathrm{Na}$ presente pesquisa, tanto o consumo de frutas, verduras e fibras foi relatado quanto a restrição no consumo de sal, doces e gorduras, o que evidencia o conhecimento dos participantes sobre o benefício e o efeito protetor da alimentação como forma de tratamento da patologia.

A restrição no uso de sal citada na pesquisa também é orientada pelas diretrizes as quais afirmam que uma dieta contendo cerca de $1 \mathrm{~g}$ de sódio promoveu rápida e importante redução de PA em hipertensos resistentes. A necessidade nutricional de sódio para os seres humanos é de $500 \mathrm{mg}$, tendo sido definida, pela Organização Mundial de Saúde, em $5 \mathrm{~g}$ de cloreto de sódio ou sal de cozinha (que corresponde a $2 \mathrm{~g}$ de sódio) a quantidade máxima considerada saudável para ingestáo alimentar por dia. O consumo médio do brasileiro corresponde ao dobro do recomendado ${ }^{13}$.

Evitar o excesso de vinho e o consumo de bebida alcoólica também foi citado pelos participantes da pesquisa como forma de tratamento. Segundo as VI Diretrizes Brasileiras de Hipertensão Arterial, essa relação está clara, pois uma quantidade maior de etanol eleva a PA e está associada a maiores morbidade e mortalidade cardiovasculares ${ }^{13}$. Pesquisa realizada por Conceição et al. demonstrou que o consumo de álcool apresentouse fortemente associado aos níveis maiores de PA, em ambos os sexos ${ }^{22}$.

O consumo inadequado de café, de chá estimulante e de chimarrão, apontado pelos participantes não é citado pelas VI Diretrizes Brasileiras de Hipertensáo Arterial, que orientam o uso moderado de café, pela presença de polifenóis com propriedades vaso protetoras, mas apontam que a elevação da (PA) é irrelevante. Estudo transversal não encontra associação entre o hábito de consumir chimarrão e a hipertensão, no entanto, encontra-se pesquisa demonstrando que a cafeína tem mostrado um aumento modesto da pressão arterial sistólica, entre $5 \mathrm{mmHg}$ e $10 \mathrm{mmHg}$, e que a taquicardia ocorre mais em indivíduos sem o hábito de consumir xantinas e é dose dependente ${ }^{23,24}$.

Em consonância com os achados da pesquisa, o Ministério da Saúde recomenda limitar o consumo de açúcar livre, açúcar de mesa, refrigerantes e sucos artificiais, doces e guloseimas em geral, reduzir o consumo de carnes gordurosas, embutidos, leite e derivados integrais, retirar a gordura aparente da carne e a pele do frango e do peixe e preferir óleos vegetais ${ }^{3}$.

Como discutido anteriormente, o cuidado com a doença envolve os sentimentos, a cultura, as crenças e os conhecimentos populares, aos quais, muitas vezes, os indivíduos apoiam-se como forma de tratamento alternativo.

O uso da folha de cana para o controle dos níveis de PA mencionado na pesquisa, também foi observado em outros estudos ${ }^{25}$. A folha de cana foi citada em cinco entrevistas como combatente de afecçôes renais e dor de estômago, podendo ser associada a outras plantas, como a folha de abacate e a folha de lima ${ }^{26}$. No entanto, não foram encontrados dados científicos que comprovem a sua eficácia, assim como não foi encontrado registro dessa planta na lista de medicamentos fitoterápicos da Agência Nacional de Vigilância Sanitária (ANVISA) ${ }^{27}$.

Da mesma maneira, não foram achadas evidências científicas que comprovem os benefícios do uso do caldo de cana. Sabe-se apenas que o caldo de cana é um alimento rico em potássio, o que pode justificar o seu uso.

Para o limão, usado como forma de tratamento, não se encontrou confirmação na literatura científica; porém seu uso deve ser aconselhado por se tratar de uma fruta e por ser fonte de vitamina C, que, em alguns estudos, vem sendo utilizada como acelerador de vasodilataçáa ${ }^{28}$.

Pesquisas demonstram que, em crianças obesas, a suplementação de vitamina $C$ pode levar a uma redução da pressão arterial e a um aumento do fluxo sanguíneo muscular, tanto em condiçóes de repouso quanto em resposta a desafios, como o estresse mental ${ }^{28}$.

Também foram levantados, durante as discussôes do seminário, outros cuidados referentes ao tratamento 
não medicamentoso que auxiliam no controle da hipertensão, como: não esquecer os remédios; não fumar; fazer caminhadas e exercício físico; procurar manter a calma, evitar o estresse.

O sedentarismo é a principal causa do aumento da incidência de várias doenças, como HAS, diabetes, obesidade, ansiedade, colesterol al to e infarto do miocárdio. A Sociedade Brasileira de Cardiologia (SBC) afirma que a atividade física reduz a incidência de HAS e que o estresse emocional participa do seu desencadeamento e pode funcionar como barreira para a adesão ao tratamento e para a mudança de hábitos ${ }^{29}$.

O exercício físico de baixa intensidade reduz a pressão arterial porque causa redução no débito cardíaco, o que pode ser explicado pela diminuição na frequência cardíaca de repouso, e redução do tônus simpático no coração, em decorrência de menor intensificação simpática e maior retirada vagal ${ }^{30}$.

O tabagismo está diretamente envolvido no desenvolvimento de doenças cardiovasculares, de maneira primária ou secundária, e a sua cessação deve ser encorajada a todos os pacientes hipertensos, pois aumenta muito o risco de complicaçóes cardiovasculares em pacientes portadores de hipertensão arterial ${ }^{13,31}$.

O cigarro não é identificado como um fator de risco para o desenvolvimento da HAS, mas se associa ao aumento da variabilidade da pressão arterial. Em estudos internacionais, $75 \%$ a $90 \%$ dos médicos oferecem conselho para cessar o uso do tabaco, embora apenas 50\% dos tabagistas relatam terem sido orientados ${ }^{11}$.

$\mathrm{O}$ processo de adesão ao tratamento anti-hipertensivo é complexo e lento, mesmo com o paciente confirmando a sua eficácia no controle da pressão alta, como referido. Trata-se de adentrar na vida da pessoa, criar novos hábitos, mudar a forma de viver e diminuir o "prazer de viver" do hipertenso ${ }^{29}$.

Os benefícios alcançados com as mudanças na alimentação e prática de atividade física não são prontamente percebidos pelos pacientes, o que os desencoraja. Não devemos esquecer que a alimentação é um dos primeiros prazeres que os seres humanos reconhecem desde o nascimento; já a atividade física necessita intencionalidade e dedicaçáo de tempo. Muitos desistem da caminhada por alegarem "sentir canseira" ou que "o resultado é apenas cansaço" ${ }^{\text {. }}$

Sales e Tamaki demonstram relatos de hipertensos segundo os quais não deixam de comer para seguir o tratamento; ainda segundo os pesquisadores, o uso de medicamento náo exige mudanças radicais no estilo de vida, nem requer alocação considerável de tempo ${ }^{8}$. Além disso, na grande maioria dos casos, os medicamentos estáo disponíveis na rede do SUS ${ }^{8}$.

Estudos demonstram que $75 \%$ dos pacientes que recebem recomendaçóes médicas relacionadas a mudanças no estilo de vida, como a restriçáo alimentar, não as seguem $^{32}$.

Os pacientes parecem se eximir da responsabilidade de buscar o controle da pressáo. Quando questionados sobre o que podem fazer para efetivar esse controle, eles, em sua maioria, apontaram mudanças nos hábitos. Todavia, existe uma lacuna entre o que os pacientes acreditam e o que realmente fazem ${ }^{33}$.

Náo conseguir preparar refeiçóes separadas para o hipertenso e o restante da família, é uma atitude que demonstra descaso de ambas as partes, pois se sabe que o fator genético pode levar um indivíduo saudável a ter hipertensão, e esse risco é ainda maior quando proporcionado pelo ambiente em que vive $e^{34}$.

Não aproveitar as frutas da época; a falta de interesse em fazer uma horta; a falta de hábito de fazer caminhada porque recebe visita à tarde ou por falta de tempo, assim como os demais argumentos usados para justificar a não adesão ao tratamento não-medicamentoso, indicam, mais uma vez, a dificuldade que os pacientes enfrentam na mudança de hábitos ${ }^{33}$.

Pode-se observar na referente pesquisa que todos os participantes realmente sabiam quais medidas deveriam ser tomadas para garantir um tratamento eficaz; todavia, os relatos de descuido por preguiça, exagero, diminuiçáo do paladar e falta de vontade de modificar hábitos alimentares e estilo de vida corroboram outros estudos $^{35}$.

Nos debates durante a exposiçẫo da plenária final, observou-se ser consenso entre os participantes que o náo seguimento dos cuidados recomendados se deve a falta de comprometimento, de disciplina, de motivação, de persistência e de paciência, requisitos considerados fundamentais para que se efetivem mudanças comportamentais significativas e duradouras, bem como para adaptação do paladar a uma alimentação hipossódica e hipolipídica, rica em frutas e verduras.

Em muitas situaçóes, pode ocorrer uma tentativa das pessoas negarem o tratamento náo farmacológico, o que poderia ser explicado pela dificuldade que muitas relatam, na prática clínica, de mudar um hábito ou um estilo de vida ${ }^{33}$. Por outro lado, os problemas financeiros e familiares foram mencionados como grande causa da não adesão ao tratamento, corroborando a assertiva de que, em alguns casos, os fatores socioeconômicos interferem na baixa adesão ao tratamento ${ }^{12}$. Nesse seguimento, o estudo realizado com mulheres aponta que o nível socioeconômico determina diferenças de consumo, sendo o baixo nível socioeconômico determinante da menor presença de legumes e frutas na dieta ${ }^{36}$.

A leitura do rótulo nutricional e a diferenciação entre diet e light identificada no estudo como causa de dificuldade em seguir a conduta dietoterápica é questionada pela Agência Nacional de Vigilância Sanitária, 
que levantou dados junto à população e demonstrou que aproximadamente $70 \%$ das pessoas consultam os rótulos no momento da compra; no entanto, mais da metade não entende adequadamente o significado das informações ${ }^{37}$.

\section{Conclusões}

O estudo permitiu observar que os entrevistados têm conhecimento sobre o tratamento não-medicamentoso e sobre o que os profissionais de saúde recomendam para o controle da pressão, porém têm dificuldade em seguir tais recomendaçóes. Essa dificuldade pode estar relacionada às grandes mudanças que se impóem nos hábitos alimentares, no estilo de vida e até mesmo nas crenças pessoais.

O fato da hipertensão se caracterizar como uma doença crônica de manifestaçáo silenciosa pode ser um dos motivos pelos quais os hipertensos não sentem a necessidade de adequaçóes ao estilo de vida, o que sugere que a preocupaçáo advém mediante sintomas. Outro fator relevante que pode ser destacado como motivo de tal descuido está relacionado ao tratamento medicamentoso, pois a crença de que a regulaçáo da pressão é obtida através de fármacos mantém, de certo modo, os hipertensos em uma zona de conforto, fazendo que não alterem seus hábitos.

Assim, nota-se a importância de novas estratégias de controle da hipertensão, a partir de meios não-medicamentosos, através de orientaçóes referentes aos efeitos colaterais das medicaçóes e à qualidade de vida, destacando os benefícios de uma alimentação saudável e uma vida ativa tanto no tratamento da hipertensão como na prevenção de suas complicaçóes e de outras doenças como diabetes mellitus e as doenças coronarianas que podem ser prevenidas por meio de um estilo de vida mais saudável.

\section{Referências}

1. Bastos DS, Borenstein MS. Identificando os déficits de autocuidado de clientes hipertensos de um centro municipal de saúde. Texto Contexto Enferm. 2004 Jan/Mar; 13(1):92-9.

2. Sociedade Brasileira de Cardiologia. V Diretrizes Brasileiras de Hipertensão Arterial. São Paulo: SBC; 2006; 9(4):122-54.

3. Brasil. Ministério da Saúde. Secretaria de Atenção à Saúde. Departamento de Atenção Básica. Hipertensão arterial sistêmica para o Sistema Único de SaúdelMinistério da Saúde. Brasília: Ministério da Saúde; 2006.

4. Organização Pan-Americana da Saúde. Linhas de cuidado: hipertensão arterial e diabetes. Brasília: Organização PanAmericana da Saúde; 2010.
5. Brasil. Inquérito domiciliar sobre comportamentos de risco e morbidade referida de doenças e agravos não transmissíveis. Brasil, 15 capitais e Distrito Federal 2002-2003. Coordenação Geral de Agravos e Doenças Não Transmissíveis, Secretaria de Vigilância em Saúde, Ministério da Saúde. [Internet]. 2003 [citado em 19 jan. 2013]. Disponível em: http://portal.saude.gov.br/portal/ saude/profissional/area.cfm?id_area $=1477$

6. Oliveira CJ, Moreira TMM. Caracterização do tratamento nâo-farmacológico de idosos portadores de hipertensão arterial. Rev Rene Fortaleza. 2010 Jan/Mar; 11(1):76-85.

7. Brasil. Ministério da Saúde. A vigilância, o controle e a prevenção das doenças crônicas não transmissíveis: DCNT no contexto do Sistema Único de Saúde brasileiro. Brasília: Organização Pan-Americana da Saúde; 2005.

8. Sales CM, Tamaki EM. Adesão às medidas de controle da hipertensão arterial sistêmica: o comportamento do hipertenso. Cogitare Enferm. 2007 Abr/Jun;12(2):157-63.

9. Cuppari L, coordenadora. Guia de nutrição: nutrição clínica no adulto. Barueri: Manole; 2002.

10. Machado IC, Kirsten VR. Adesão ao tratamento nutricional de pacientes adultos atendidos em uma clínica de Santa MariaRS. Disc Scientia, Série: Ciênc Saúde. 2011;12(1): 81-91.

11. Gasperin D, Fensterseifer LM. As modificaçóes do estilo de vida para hipertensos. Rev Gaúcha Enferm. 2006 Set; 27(3):372.

12. Maciel EAM. A não-adesão ao tratamento da hipertensão por pacientes trabalhadores. [Dissertação]. Brumadinho: Universidade Federal de Minas Gerais; 2012.

13. 13. Sociedade Brasileira de Cardiologia. VI Diretrizes Brasileiras de Hipertensão Arterial. Arq Bras Cardiol. 2010: $1-51$.

14. Lopes LO, Moraes ED. Tratamento não medicamentoso para hipertensão arterial. ACTA Brasileira de Pesquisa em Saúde [Internet]. 2011 Out/Dez [citado em: 13 ago. 2013]; 8(1):1-8. Disponível em: http://www.inesul.edu.br/revista_saude/arquivos/arq-idvol_10_1339682941.pdf

15. Gusmão JS, Mion D. Adesão ao tratamento - conceitos. Rev Bras Hipertens. 2006; 13 (1):23.

16. Barbosa RBG, Lima, NKC. Índices de adesão ao tratamento anti-hipertensivo no Brasil e mundo. Rev Bras Hipertens. 2006; 13 (1): 35.

17. Santos AJM, Rosa C, Oliveira EL, Almeida JR, Schneider RM, Rocha SSL, Coutinho RMC. A não adesão de pacientes hipertensos ao tratamento em uma Unidade Básica de Saúde. Rev Bras Hipertens. 2009; 13 (1):35.

18. Minayo MCS. O desafio do conhecimento: pesquisa qualitativa em saúde. $7^{a}$ ed. São Paulo; Rio de Janeiro:Hucitec; Abrasco; 2009.

19. Brasil, Ministério da Saúde. Resolução no 466, Conselho Nacional de Saúde; 2012.

20. Silva FVF, Silva LF, Guedes MVC, Moreira TMM, Rabelo ACS, Ponte KMA. Cuidado de enfermagem a pessoas com hipertensão fundamentado na teoria de Parse. Esc Anna Nery 
[Internet].2013;Jan/Mar[citadoem 17jul.2013];17(1):1119. Disponível em: http://www.scielo.br/scielo. php?script=sci_arttext $\&$ pid $=$ S1414-1452013000100016

21. Girotto E, Andrade SM, Cabrera MAS, Matsuo T. Adesão ao tratamento farmacológico e não farmacológico e fatores associados na atenção primária da hipertensão arterial. Ciência \& Saúde Coletiva [Internet]. 2013 [citado em 10 ago. 2013];18(6):1763-72. Disponível em: http://www. scielo.br/scielo.php?script=sci_arttext $\& \operatorname{lng}=$ pt $\&$ nrm $=$ iso\&tlng=pt\&pid=S1413-81232013000600027

22. Conceição TV, Gomes FA, Tauil PL, Rosa TT. Valores de pressão arterial e suas associações com fatores de risco cardiovasculares em servidores da Universidade de Brasília. Arquivos Brasileiros de Cardiologia [Internet]. 2006 Jan [citado em 10 ago. 2013]; 86(1):26-31. Disponível em: http:// www.scielo.br/pdf/\%0D/abc/v86n1/a05v86n1.pdf

23. Hartmann M, Dias-da-Costa JS, Olinto MTA, Pattussi MP, Tramontini A. Prevalência de hipertensão arterial sistêmica e fatores associados: um estudo de base populacional em mulheres no sul do Brasil. Cad. Saúde Pública [Internet]. 2007 Ago [citado em 15 jul. 2013]; 23(8):1857-66. Disponível em: http://www.scielo.br/pdf/csp/v23n8/12.pdf

24. Poerschke RA, Lisboa HRK. Efeitos da ingestáo aguda de chimarrão (Ilex paraguariensis St. Hil) na função endotelial e nos sinais vitais. [Dissertação] [Internet] Porto Alegre: Universidade Federal do Rio Grande do Sul, 2009. [citado em 11 ago. 2013]. Disponível em: http://www.lume.ufrgs. $\mathrm{br} /$ handle/10183/16858

25. Pilla MAC, Amorozo CM, Furlan A. Obtenção e uso das plantas medicinais no distrito de Martim Francisco, município de Mogi-Mirim, SP, Brasil. Acta Bot Bras, 2006; 20(4):789-802.

26. Rezende HA, Cocco MIM. A utilização da fototerapia no cotidiano de uma população rural. Rev Esc Enferm USP. 2002;36(3):282-8.

27. Brasil. Agência Nacional de Vigilância Sanitária. Instrução Normativa no 05 [Internet]. Brasília: Diretoria Colegiada; 2008 [citado em 03 jul. 2013]. Disponível em: http://portal.saude.gov.br/portal/arquivos/pdf/IN_N_5_2008_anvisa.pdf

28. Fernandes PROF. Vitamina C restaura pressão arterial e a resposta vasodilatadora no antebraço de crianças obesas. Arq Bras Cardiol. [Internet]. 2011 Mai [citado em 20 jul. 2013]; 96(6):490-7. Disponível em: http://www.scielo.br/scielo.php?script=sci_arttext\&pi$\mathrm{d}=$ S0066-782X2011000600010\&lang=pt
29. Dourado CS, Macêdo-Costa KNF, Oliveira JS, Leadebal ODCP, Silva GRF. Adesão ao tratamento de idosos com hipertensão em uma unidade básica de saúde de João Pessoa, estado da Paraíba. Maringá. 2011; 33(1):9-17.

30. Negrão CE, Rondon MUPB, Kuniyoshi FHS, Lima EG. Aspectos do treinamento físico na prevenção da hipertensão arterial. Rev Bras Hipertens. 2001; 4(3):84-7.

31. Forjaz CLM, Rezk CC, Melo CM, Santos DA, Teixeira L, Nery SS, Tinucci T. Exercício resistido para o paciente hipertenso: indicação ou contraindicação. Rev Bras Hipertens. 2003; 10(2):119-24.

32. Koehnlein EA, Salado GA, Yamada NA. Adesão à reeducação alimentar para perda de peso: determinantes, resultados e a percepção do paciente. Rev Bras Nutr Clin [Internet]. 2008 [citado em 18 ago. 2013]; 23(1):56-65. Disponível em: http://www.ucg.br/ucg/eventos/obesidade_curso_capacitacao_ambulatorial/Material_Consulta/Material_ Nutricao/Ades\%C3\%A3o\%20\%C3\%A0\%20reeduca\%C3\%A7\%C3\%A3o\%20alimentar\%20para\%20perda\%20 de $\% 20$ peso.pdf

33. Péres DS, Magna JM, Viana LA. Portador de hipertensão arterial: atitudes, crenças, percepçóes, pensamentos e práticas. Rev Saúde Pública [Internet]. 2003 [citado em 10 ago. 2013]; 37(5):635-42. Disponível em: http://www.scielo.br/scielo. php?script=sci_arttext\&pid=S0034-89102003000500014

34. Salgado CM, Carvalhes JTA. Hipertensão arterial na infância. J. Pediatr. 2003; 79(1):115-24.

35. Callejon KS, Paternez ACAC. Adesão ao tratamento nutricional por pacientes atendidos na clínica de nutrição docente-assistencial da Universidade Municipal de São Caetano do Sul (USCS). Ver Bras Ciên Saúde [Internet]. 2008 Jul/ Set; 6,(17):3-8. Disponível em: http://seer.uscs.edu.br/index.php/revista_ciencias_saude/article/view/353/171

36. Jorge MIE, Martins IS, Araujo EAC. Diferenciais socioeconômicos e comportamentais no consumo de hortaliças e frutas em mulheres residentes em município da região metropolitana de São Paulo. Rev Nutr [Internet]. 2008 Nov/Dez [citado em 15 jul. 2013]; 21(6):695703. Disponível em: http://www.scielo.br/scielo. php?pid=S1415-52732008000600008\&script=sci_arttext

37. Brasil, Ministério da Saúde. Rotulagem nutricional obrigatória: manual de orientaçáo aos consumidores [Internet]. Brasília: Agência Nacional de Vigilância Sanitária; Universidade de Brasília; 2005 [citado em 10 ago 2013]. Disponível em: http://www.anvisa.gov.br/alimentos/rotulos/manual_consumidor.pdf. 